\title{
Educação em saúde como estratégia para a adesão ao autocuidado e às práticas de saúde em uma unidade de saúde da família
}

\author{
Education in healthcare as a strategy for self-care adherence and health \\ practices in a family health unit
}

\author{
Gabriel Fernandes de Sousa ${ }^{1}$, Karolinny Donato Pinto de Oliveira ${ }^{1}$, \\ Sandra Maria Dias de Queiroz ${ }^{2}$
}

Sousa GF, Oliveira KDP, Queiroz SMD. Educação em saúde como estratégia para a adesão ao autocuidado e às práticas de saúde em uma unidade de saúde da família / Education in healthcare as a strategy for self-care adherence and health practices in a family health unit. Rev Med (São Paulo). 2019 jan.-fev.;98(1):30-9.

RESUMO: Objetivo: Promover uma melhor adesão ao autocuidado e às práticas de saúde, estimulando a participação ativa dos usuários nas atividades realizadas pela Unidade de Saúde da Família (USF) Camalaú. Metodologia: Estudo descritivo desenvolvido a partir de um projeto de intervenção elaborado no Internato de Medicina de Família e Comunidade do curso de Graduação em Medicina em uma instituição de ensino. Foram realizadas palestras e rodas de conversa que enfatizaram a importância do autocuidado e sua interferência na comunidade no âmbito pessoal e da saúde. O público selecionado foi constituído pelos usuários presentes na sala de espera da unidade durante o momento das atividades. A análise dos dados foi realizada com os profissionais de saúde nas reuniões semanais de equipe. Resultados e Discussão: Foi constatada considerável melhora e progresso na adesão ao autocuidado e compreensão do processo saúde-doença e maior nível de conhecimento da população sobre as doenças mais prevalentes da comunidade e suas possíveis complicações. Após a realização das ações, as condutas possuíam maior nível de aceitabilidade. Conclusão: A educação em saúde tem grande importância para que a USF assegure integralmente a promoção à saúde e as reuniões de equipe são essenciais neste processo. Essa intervenção, a longo prazo, possivelmente contribuirá para uma melhora significativa na terapêutica dos usuários, no controle e prevenção de doenças e favorecerá uma melhor qualidade de vida para a comunidade.

Descritores: Educação em saúde; Autocuidado; Atenção primária à saúde; Promoção da saúde.

\begin{abstract}
Objective: To promote a better adherence to selfcare and health practices, stimulating the active participation of users in the activities carried out by the Camalaú Family Health Unit (FHU). Methodology: A descriptive study developed from an intervention project elaborated at the Internship of Family and Community Medicine of the undergraduate course in Medicine in a teaching institution. Lectures and round of conversations were held, emphasizing the importance of self-care and its influence in the community, both in the personal and health care spheres. The selected public was constituted by the users present in the waiting room of the unit during the moment of the activities. Data analysis was performed with health professionals at weekly team meetings. Results and Discussion: There was a considerable improvement and progress in the adherence of self-care and in understanding the health-disease process, and a higher level of knowledge of the population on the most prevalent diseases in the community and their possible complications. After those actions, the conducts had a higher level of acceptability. Conclusion: Education in Health Care is of great importance for FHU to fully ensure health promotion, and team meetings are essential in that process. This intervention, in the long term, will possibly contribute to a significant improvement in the therapeutics of users, in the control and prevention of diseases and will favor a better quality of life for the community.
\end{abstract}

Keywords: Health education; Self-care; Primary health care; Health promotion.

1. Faculdade de Ciências Médicas da Paraíba (FCM-PB). Acadêmico(a) do curso de graduação em Medicina da FCM-PB. ORCID: Sousa GF - https://orcid.org/0000-0002-8923-1475. Oliveira KDP - https://orcid.org/0000-0002-4007-4114. E-mail: gabrielfernanddes@ hotmail.com; karolinnydonato@hotmail.com.

2. Faculdade de Ciências Médicas da Paraíba (FCM-PB). Farmacêutica/Mestre em Educação e Professora do curso de graduação em Medicina da FCM-PB. ORCID: https://orcid.org/0000-0003-2277-6099. E-mail: queirozmaria1010@gmail.com.

Endereço para correspondência: Gabriel Fernandes de Sousa. Faculdade de Ciências Médicas da Paraíba. BR 230 - KM 09, Intermares, Cabedelo, Paraíba, Brasil. E-mail: gabrielfernanddes@hotmail.com. 


\section{INTRODUÇÃO} autocuidado é uma atividade exercida e
assimilada pelo indivíduo com o propósito de ser orientada para um objetivo. É uma ação desenvolvida em situações concretas da vida, na qual a pessoa a dirige para si mesma, ou para regular os fatores que afetam seu próprio desenvolvimento, atividades em benefício da vida, saúde e bem estar. Ele está centrado no paradigma da totalidade e adota o pressuposto de que o ser humano é o conjunto de suas partes, sendo a soma do biológico, psicológico, espiritual e social ${ }^{1}$. É considerado um processo pelo qual uma pessoa leiga atua em seu próprio interesse na promoção da sua saúde, na prevenção e detecção da doença e no seu tratamento a nível da atenção básica em saúde².

Criado em 1994 pelo Ministério da Saúde, o Programa Saúde da Família (PSF) é uma proposta de reorientação do modelo assistencial brasileiro ${ }^{3}$, tendo seu enfoque voltado para a relação com a comunidade, assumindo o compromisso de prestar assistência universal, integral, equânime, contínua e resolutiva à população, em conformidade com suas reais necessidades. Busca, todavia, a satisfação do usuário através do estreito relacionamento dos profissionais com o indivíduo, família e comunidade ${ }^{4,5}$.

Portanto, o autocuidar é uma habilidade adquirida que identifica as necessidades e assistência ao indivíduo, regula os processos vitais, mantém e promove a atividade, o desenvolvimento e a integridade do organismo, gerando, a partir disso, o bem estar ${ }^{1}$. Dessa forma, sua prática deve ser incentivada pelos profissionais de saúde da família na comunidade a qual estão inseridos. Perante o exposto, este estudo justificou-se pela importância do autocuidar e da adesão às práticas de saúde na melhora da qualidade de vida da população, evitando agravamentos e complicações de doenças e execução da prevenção em saúde.

Após a realização de reunião com a equipe de saúde da Unidade de Saúde da Família (USF) Camalaú, foram levantadas as principais problemáticas existentes e observou-se que existia grande resistência dos usuários na participação das atividades realizadas devido à baixa adesão ao autocuidado e às práticas de saúde. Havia baixa frequência na participação da comunidade nos grupos de apoio e nas atividades educativas, mesmo com a existência de profissionais capacitados. Além disso, foi relatado pela equipe de saúde que alguns grupos de apoio encerraram suas atividades pela baixa assiduidade da comunidade. A partir dessas informações, foi verificada a importância dos usuários possuírem ciência do que realmente precisam para que tenham um cuidado integral e multidisciplinar adequado e saciem essa necessidade usufruindo das atividades que a própria unidade de saúde tem a oferecer.

Logo, este estudo objetivou promover uma melhor adesão ao autocuidado e às práticas de saúde, estimulando a participação ativa dos usuários nas atividades realizadas pela USF Camalaú, visando aprimorar a qualidade de vida da população.

\section{MÉTODOS}

Trata-se de um estudo descritivo, elaborado no contexto do curso de Graduação em Medicina da Faculdade de Ciências Médicas da Paraíba, desenvolvido a partir de um projeto de intervenção realizado durante o Internato de Medicina de Família e Comunidade, sendo uma atividade obrigatória para a conclusão do estágio curricular. A USF do referido estudo localiza-se no bairro de Camalaú que pertencente ao município de Cabedelo-PB. O público selecionado foi constituído pelos usuários presentes na sala de espera da unidade durante o momento da realização das atividades, sendo composto principalmente por hipertensos, diabéticos, gestantes, idosos, mulheres adultas e poucos homens adultos. As datas foram selecionadas de acordo com o enfoque dado às consultas, pelos profissionais de saúde, conforme agenda pré-estabelecida da USF. A análise dos dados foi realizada com os profissionais de saúde nas reuniões semanais de equipe.

Para a realização, inicialmente, foi constatada a problemática supracitada juntamente com os profissionais de saúde da USF através de uma reunião com a equipe de saúde da unidade, onde foram levantadas as dificuldades e desafios para uma melhor promoção da saúde na comunidade. Foi acordada a realização de palestras e rodas de conversa que durassem cerca de trinta minutos e que enfatizassem a importância do autocuidado e como isso poderia interferir na vida dos pacientes, no âmbito pessoal e da saúde, as quais foram realizadas em dias estratégicos, de acordo com a programação semanal da USF. Foi construído um cronograma de ações para um melhor planejamento da execução dessas atividades. Além disso, também foram abordadas as principais patologias existentes na comunidade e suas complicações, enfatizando os métodos preventivos necessários. Também foram esclarecidas quais eram as medidas não farmacológicas mais importantes e como incluí-las no dia-a-dia.

Nas palestras dialogadas seguidas de rodas de conversa foram convidados alguns profissionais de saúde da unidade, como o médico, enfermeira, fisioterapeuta e nutricionista, o que contribuiu para uma melhor compreensão dos temas abordados. Ademais, foi estimulada a participação ativa dos usuários, reservando alguns momentos para questionamentos e sugestões. Foram confeccionados panfletos (Figura 1) contendo a programação semanal da unidade, quais os profissionais e serviços que a USF disponibiliza e quais grupos de apoio e atividades são realizadas semanalmente para aprimorar o cuidado dos usuários. Esse material foi distribuído na sala de espera da unidade, nas visitas domiciliares e também pelos agentes comunitários de saúde. Ao final das palestras, o atendimento era iniciado e, durante sua realização, os usuários eram questionados sobre o impacto gerado em suas vidas, sendo reforçadas algumas informações quando observada a persistência de dúvidas. 
As estratégias educativas adotadas, as rodas de conversas e as palestras dialogadas se fundamentaram na perspectiva da educação libertadora de Paulo Freire por ser uma valiosa ferramenta metodológica para o desenvolvimento das ações de educação em saúde. Paulo Freire, declarado Patrono da Educação Brasileira em 2012, foi um pioneiro do trabalho de sistematização teórica da Educação Popular, cujos princípios e práticas inspiraram o movimento da educação popular em saúde, esse surgido na década de 70 do século passado. Entre os princípios da Pedagogia Freiriana está o de compreender a educação como ato político na medida que facilita processos de crítica e autocrítica dos sujeitos no enfretamento dos seus problemas ${ }^{6 ; 7 ; 8}$, com vistas à transformação desses na luta por direitos e cidadania. Outro princípio é o do diálogo como mediação para essa transformação. A partir das conversas sobre os conflitos e do cruzamento de pensamentos, o método de rodas de conversa e de palestras dialogadas foi compreendido como uma ação pedagógicatransformadora ${ }^{9,10}$.

\section{UNIDADE DE SAÚDE DA FAMÍLIA (NOME DA UNIDADE)}

- PROFISSIONAIS DISPONÍVEIS PELA USF E PELO NASF

\begin{tabular}{|c|c|}
\hline MÉDICOS & Nome dos(as) médicos(as) e suas especialidades \\
\hline ENFERMEIRA & Nome do(a) enfermeiro(a) \\
\hline DENTISTA & Nome do(a) dentista \\
\hline NUTRICIONISTA & Nome do(a) nutricionista \\
\hline FISIOTERAPEUTA & Nome do(a) fisioterapeuta \\
\hline PSICÓLOGA & Nome do(a) psicólogo(a) \\
\hline FONOAUDIÓLOGA & Nome do(a) fonoaudiólogo(a) \\
\hline ASSISTENTE SOCIAL & Nome do(a) assistente social \\
\hline EDUCADOR FísICO & Nome do(a) educador(a) físico(a) \\
\hline \multicolumn{2}{|l|}{ - GRUPOS DE APOIO } \\
\hline Educação Física & Terças e Quintas (Manhã) \\
\hline Saúde Mental & Quintas 15/15 dias (Manhã) \\
\hline Gestantes & Quartas (Manhã) \\
\hline Hipertensão e Diabetes & Terças (Tarde) \\
\hline Artesanato & Terças e Quintas (Tarde) \\
\hline Adolescentes & Terças (Tarde) \\
\hline
\end{tabular}

\section{- agenda Semanal de ATENDimento}

\begin{tabular}{|c|c|}
\hline SEGUNDA-FEIRA & $\begin{array}{l}\text { MÉDICO: Atendimento geral (Manhã e Tarde) } \\
\text { ENFERMEIRA: Puericultura (Manhã) e Atendimento geral (Tarde) } \\
\text { DENTISTA: Atendimento infantil (Manhã) e adulto (Tarde) } \\
\text { NUTRICIONISTA: Puericultura (Manhã) } \\
\text { FISIOTERAPEUTA: Puericultura (Manhã) } \\
\text { FONOAUDIÓLOGA: Puericultura e Atendimento geral (Manhã) } \\
\text { HOMEOPATA: Consultas agendadas (Tarde) }\end{array}$ \\
\hline TERÇA-FEIRA & $\begin{array}{l}\text { MÉDICO: Hipertensão, Diabetes e Saúde mental (Manhã e Tarde) } \\
\text { ENFERMEIRA: Visita domiciliar (Manhã) e Atendimento geral (Tarde) } \\
\text { DENTISTA: Hipertensos e Diabéticos (Manhã) e infantil (Tarde) } \\
\text { NUTRICIONISTA: Atendimento geral (Manhã) }\end{array}$ \\
\hline QUARTA-FEIRA & $\begin{array}{l}\text { MÉDIIC: Pré-natal (Manhã) e Atendimento geral (Tarde) } \\
\text { EEFERMEIRA Pré-natal (Manhã) e Atendimento geral (Tarde) } \\
\text { DENTISTA: Pré-natal (Manhã) e Atendimento na Escola/Visita (Tarde) } \\
\text { NUTRICIONISTA: Pré-natal (Manhã) e Atendimento Bolsa Famillia (Tarde) } \\
\text { ASSISTENTE SOCIAL: Atendimento geral (Manhã) }\end{array}$ \\
\hline QUINTA-FEIRA & $\begin{array}{l}\text { MÉDICO: Grupo Saúde Mental/Visita (Manhãa) e Atendimento geral (Tarde) } \\
\text { PEDIATRA: Atendimento infantil (Manhāa) } \\
\text { DENTISTA: Atendimento adulto (Manhã) e infantil (Tarde) } \\
\text { FISIOTERAPEUTA: Grupo de Educação Fisica (Manhã) } \\
\text { PSICóLOGA: Grupo de Saúde Mental/ Atendimento geral (Manhã) }\end{array}$ \\
\hline SEXTA-FEIRA & ENFERMEIRA: Exame Citológico (Manhã) e Atendimento geral (Tarde) \\
\hline
\end{tabular}

Fonte: Elaborado pelos autores.

Figura 1. Modelo do panfleto distribuído na USF durante as atividades

\section{RESULTADOS E DISCUSSÃO}

Os usuários que efetivamente frequentam a USF Camalaú são constituídos principalmente por: idosos (predominantemente mulheres), mulheres adultas, gestantes, homens adultos, esses em menor proporção, e crianças, havendo baixa frequência de adolescentes e jovens de ambos os sexos. Segundo relatos dos profissionais, a unidade não possui um quantitativo da população assistida nem um banco de dados com tais informações, o que impossibilitou a inclusão desses dados neste estudo. A USF dispõe de dezoito profissionais de saúde, sendo eles: três médicos, uma enfermeira, uma dentista, uma fisioterapeuta, uma nutricionista, uma psicóloga, uma fonoaudióloga, uma assistente social, um educador físico e sete agentes comunitários de saúde (ACS).

Após a execução das ações, foi detectada considerável melhora e progresso diante da aceitabilidade, por parte dos usuários presentes nas palestras e rodas de conversas ministradas, na adesão ao autocuidado e compreensão do processo saúde-doença. Os objetivos propostos foram cumpridos, ou seja, a população foi incentivada quanto aos hábitos e estilo de vida saudáveis; foram confeccionados e distribuídos panfletos contendo a organização e serviços disponíveis na USF Camalaú; elevou-se o nível de conhecimento da população sobre as doenças mais prevalentes da comunidade e suas possíveis complicações; foram expostas as principais dificuldades e desafios da USF na promoção da saúde na comunidade; os usuários foram inseridos nos grupos de apoio presentes na unidade; foram esclarecidos o papel e função dos profissionais de saúde; estimulou-se a adesão dos pacientes ao seu tratamento e foram promovidas palestras e rodas de conversa lúdicas, as quais enfatizaram a importância do autocuidado em saúde, estimulando mudanças no cotidiano capazes de gerar benefícios à saúde.

Aproximadamente $80 \%$ dos usuários que vão ao atendimento o fazem apenas com o intuito de renovar a receita de seus medicamentos de uso contínuo, possuindo baixa adesão às medidas terapêuticas não farmacológicas, as quais também garantem um impacto relevante no tratamento, como: a realização regular de atividade física, controle pressórico e glicêmico continuados, acompanhamento psicológico adequado e a adesão à uma dieta equilibrada, de acordo com a necessidade individual. Para tal população, as medidas que não eram de cunho farmacológico se encontravam na periferia do tratamento, muitas vezes nem sendo cogitadas como essenciais, pois não havia, por parte dos usuários, uma percepção de integralidade e multidisciplinaridade que exigisse o autocuidado. As limitações das pessoas para se comprometerem às ações do autocuidado estão associadas com a subjetividade do indivíduo ou em vias de envelhecimento, das ações relacionadas ou derivadas da saúde, que as capacitam parcial ou totalmente para conhecer os requisitos existentes ou emergentes da assistência reguladora das mesmas ${ }^{1}$. Logo, o que não os fazia tomar para si, portanto, a consciência de que, para gerar saúde, exige-se a participação ativa do paciente em seu próprio 
Sousa GF, et al. Educação em saúde como estratégia para a adesão ao autocuidado e às práticas de saúde.

tratamento e que o envolvimento nas atividades realizadas, pela própria unidade de saúde, é uma das maneiras de promover o autocuidado.

Historicamente falando, o cuidado da saúde fazia parte do cuidado que as pessoas tinham do cuidar de si, da sua família e da sua comunidade ${ }^{11}$. Autor seminal da área da qualidade do cuidado, Donabedian ${ }^{12}$ definiu cuidado de qualidade como aquele capaz de maximizar o bem-estar do paciente, após se levar em conta o balanço entre os ganhos e perdas esperados em todas as etapas do processo. Quando os indivíduos tomam essa conscientização de que precisam desenvolver o seu próprio processo de cuidado para que gerem saúde para si, tudo o que concerne ao tratamento imposto torna-se não uma mera obrigação a ser cumprida, mas zelo diante de sua própria vida.

O observado na comunidade foi justamente o oposto, isto é, pessoas que não entendiam a relevância de estarem atentas aos sinais de alerta que seu próprio corpo evidencia, ou de prevenirem a manifestação de tais sintomas antes da doença efetiva através de uma prevenção eficaz, acabando, por consequência, fadadas à medicalização sem ao menos terem o interesse em compreender o porquê de sua terapia, aceitando que sua melhora, controle ou cura estivessem restritas apenas ao uso dos fármacos, ressignificando o papel dos medicamentos no seu tratamento e construindo formas particulares de lidar com sua condição de saúde e as recomendações profissionais que recebem ${ }^{13}$. Dessa forma, cabe ao profissional de saúde identificar entre os pacientes as barreiras que podem levá-los ao não cumprimento do tratamento e oferecer as informações e condições necessárias para que ele compreendam a racionalidade terapêutica da prescrição apresentada e a forma mais adequada de utilizar os medicamentos recomendados ${ }^{13}$.

Um estudo brasileiro aponta as mulheres como maiores detentoras de conhecimentos e tratamento em relação aos homens. Mas, ainda assim, são mal cuidadas e se tornam fonte de problemas, pois a equipe de saúde da família não consegue controlar o cuidado individual ${ }^{14}$. Uma das problemáticas observadas e evidenciadas pelos profissionais que trabalham na unidade de saúde em Camalaú foi exatamente essa, usuárias que em sua maioria até buscam o serviço, mas apenas para renovação de receitas e solicitações de exames, sendo raras aquelas que mantinham um programa de cuidados que perpassasse o tratamento medicamentoso. Alguns pacientes chegavam a ficar revoltados quando não eram prescritos exames ou quando não eram encaminhados para um especialista, sendo que a conduta mais importante no momento seria uma intervenção multidisciplinar com um nutricionista ou fisioterapeuta, por exemplo. Eles acreditavam que o acompanhamento periódico por esses profissionais era enfadonho e não resolutivo, o que podia ser justificado pela escassez de informações sobre a importância do autocuidado e das terapias não farmacológicas.

A melhoria de qualidade no cuidado de saúde traduz- se em mudanças que produzem, direta ou indiretamente, melhores resultados de saúde, incorporando elementos técnicos passíveis de certo grau de padronização, mas, principalmente, interações pessoais fortemente imbrincadas com o contexto ${ }^{15}$. A USF Camalaú conta com estratégias de cuidado à população que visam humanizar as práticas de saúde, buscando a satisfação do usuário através do estreito relacionamento dos profissionais com a comunidade, aprimorando o processo de promoção à saúde ${ }^{5}$, como ocorre com os grupos de apoio.

A tecnologia de grupo de apoio/suporte é um recurso que vem sendo usado por profissionais de saúde, pois os auxilia a aliviar sentimentos de solidão e isolamento social, possibilitando troca de experiências e reflexão. A utilização de grupos de suporte/apoio requer a criação de um ambiente em que seus integrantes possam compartilhar suas experiências e sentimentos com a certeza de serem compreendidos pelos outros participantes ${ }^{16}$. Foi extremamente importante para os usuários entenderem o valor desses grupos, e que eles não se enquadravam em mais uma de suas obrigações da semana, mas sim em um processo de cuidado gerenciado, dinâmico e autônomo.

Alguns grupos de apoio da USF Camalaú são: Gestantes, Hipertensos e Diabéticos e Adolescentes. Para as gestantes, participar do grupo possibilita o conhecimento necessário para proceder de forma adequada diante do processo de gravidez, promovendo entendimento do que acontece em cada etapa e do que é necessário fazer para se ter uma gestação tranquila. Para os hipertensos e diabéticos, o que o grupo propõe é disseminar o entendimento de sua doença, comorbidades associadas, medidas que precisam ser tomadas para se estabelecer o controle devido e compreensão das medicações impostas e de como elas atuam. Para os adolescentes, o grupo orienta quanto às questões inerentes dessa fase atreladas à saúde, como menarca, início das atividades sexuais, alterações corporais por efeito dos hormônios, entre outras, trazendo para eles esclarecimentos e possibilidades de promoção de saúde com condutas adequadas e bem orientadas pelos profissionais certos, pois em meio a uma sociedade bombardeada de informações, que em muitos casos não são verdadeiras, faz-se necessário um repasse do que é correto e comprovado cientificamente de maneira clara e objetiva.

Além dos grupos acima citados, os usuários também têm a oportunidade de participar de grupos de educação física e de saúde mental, sendo esses, atualmente, os grupos mais frequentados na unidade, mas que ainda carecem de mais adeptos. Portanto, esta proposta de intervenção utilizou de ferramentas técnicas, por meio da análise situacional vivenciada pela unidade de saúde em Camalaú, e de atividades de caráter educativo, através de embasamento teórico científico, proporcionando à comunidade o entendimento do que significam as práticas de saúde e como elas vão além de uma consulta ou de uma medicação, sendo necessário o envolvimento e comprometimento continuado por parte dos usuários. 


\section{Primeira Atividade}

A primeira palestra ministrada aos usuários da USF Camalaú teve como temática a tuberculose e foi planejada a pedido da enfermeira da unidade, visto que é uma patologia prevalente na comunidade, mas que ainda há grande desconhecimento sobre o tema. Recebeu o título de "Tuberculose: o que é e como prevenir". Foi realizada na tarde do dia 28/03/2018 (Figura 2) na sala de espera do referido local e contou com a participação de vinte e dois usuários. A tuberculose (TB) é considerada uma condição crônica transmissível de tratamento longo, tendo como principais dificuldades para a obtenção da cura a não adesão ou abandono do tratamento, sendo o controle da doença considerado responsabilidade dos municípios e competência da Atenção Básica ${ }^{17}$. Considerando essa concepção, a palestra trouxe à tona para os usuários a importância de se conhecer mais a respeito da doença e de como ela afeta o organismo, acarretando consequências para aqueles que não efetuam um tratamento adequado e preciso diante de tal condição de saúde.

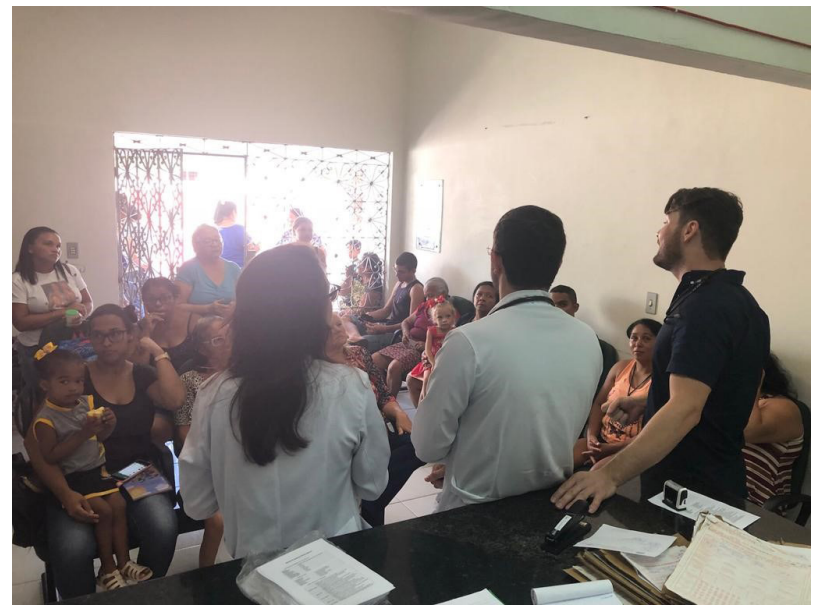

Figura 2. Realização da primeira palestra na USF

A pessoa diagnosticada com TB que recebe informação detalhada sobre a doença, bem como sobre a importância de realizar o tratamento para obtenção da cura, as reações adversas potenciais e as consequências da irregularidade do tratamento tem maior propensão à adesão ao tratamento ${ }^{17}$. A partir disso, fica evidente que quando a informação é repassada à população de forma eficaz o objetivo proposto é alcançado, tornando mais fácil realizar o tratamento naqueles que já se encontram enfermos e evitando que os contactantes, ou mesmo aqueles que estão fora da zona de risco de infecção, exerçam para si o autocuidado necessário à prevenção da doença.

Quando iniciada a palestra, foi explicado o que é a doença e de que forma ela se apresenta no ser humano. A TB é uma doença infecto-contagiosa causada pelo Mycobacterium tuberculosis ou Bacilo de Koch (BK). A doença apresenta algumas características marcantes como: um longo período de latência entre a infecção inicial e a apresentação clínica da doença; preferência pelos pulmões, mas também pode ocorrer em outros órgãos do corpo como ossos, rins e meninges; e resposta granulomatosa associada à intensa inflamação e lesão tissular ${ }^{18}$. Nessa etapa inicial, houve o direcionamento para se explicitar pontos da doença que provavelmente eram incomuns no entendimento dos usuários, como por exemplo, o fato de que a tuberculose não afeta apenas os pulmões e que é necessário receber um tratamento direcionado para cada tipo de afecção. Dessa forma, os usuários puderam sanar suas dúvidas e serem esclarecidos quanto a importantes e imprescindíveis características da doença em questão.

Além disso, a palestra foi fundamental para explicar como o diagnóstico da doença é feito e quais são os procedimentos a serem realizados após isso pelo acometido e pela equipe de saúde da família, a citar, os exames solicitados e notificação; foi demonstrado, através de dispositivo encontrado na unidade, como a baciloscopia do escarro, que essa deve ser executada pelo doente para a correta realização e resultado do exame. A principal fonte de infecção é o indivíduo com a forma pulmonar da doença, que elimina bacilos para o exterior. Estima-se que a pessoa que apresenta esse quadro pode infectar de 10 a 15 pessoas da sua comunidade num período de um ano ${ }^{18}$. Por fim, houve a explicação de como o tratamento é realizado, o porquê de ele ser executado no tempo preconizado e de que maneira ele deve ser corretamente cumprido para que se considere o paciente como tratado e curado da doença.

A principal finalidade em realizar essa palestra foi alertar os usuários em como eles devem proceder diante de uma doença tão séria e de importante grau de disseminação e infecção, compreendendo de fato que as suas ações são preponderantes para que potenciais agravantes sejam evitados, ou seja, gerando de fato o senso de prevenção na referida comunidade. Falar sobre tuberculose de forma aberta, e fora do contexto de consulta médica, faz com que o conhecimento sobre essa temática não seja limitado ou compelido apenas ao profissional de saúde, mas de que aquele que está doente ou próximo a ele também tenha um grau de responsabilidade diante do quadro e que precisa ser exercido, melhorando assim o prognóstico da doença e potencializando as chances de cura por fazer o paciente compreender que ele deve ser voz ativa no processo de adesão ao tratamento e que toda a equipe de saúde está a serviço e disposição para auxiliá-lo e conduzi-lo em sua reabilitação, sendo essa a meta proposta nesse estudo, estimular a reflexão, orientação e percepção dos usuários quanto a sua própria saúde.

O intercâmbio de saberes produz e reproduz espaços de encontro entre pessoas e instituições para reflexão, criação e ação, partindo do reconhecimento das diferenças de cada participante. O estabelecimento de vínculo, responsabilização e participação popular é fundamental para a organização do processo de trabalho centrado no 
cidadão, capaz de gerar atitudes construtivas e democráticas na mediação dos conflitos presentes no convívio entre atores sociais diversos que detêm premissas e valores diferenciados. As ações que privilegiam as pessoas, os sujeitos, são capazes de construir uma sociabilidade solidária, onde o espaço coletivo democrático pode ser usado para expressar e reconstruir os interesses comuns, debatendo problemas e tomando deliberações, sustentando a existência de uma prática transformadora, dialética, entre a práxis, a reflexão e a capacidade de escuta e de análise ${ }^{19}$.

Diante do exposto à comunidade, foi percebido que a maioria dos usuários demonstrou interesse em captar a mensagem que estava sendo transmitida a eles, e que, a partir disso, um diferencial quanto à promoção de saúde foi imposto na unidade básica de saúde em Camalaú, permitindo que o objetivo do estudo fosse seguido e que as demais palestras que se sucederiam fossem ministradas, cada qual com sua temática específica para abordar assuntos relevantes à saúde em geral e em questões particulares da população em foco, crendo que todas trariam o resultado final de melhoria na adesão ao tratamento e concepção quanto atuantes no processo saúde-doença.

\section{Segunda Atividade}

A segunda palestra recebeu o título "Como ter uma boa saúde mental?" e foi ministrada também na sala de espera da unidade básica de saúde em Camalaú, no dia 04/04/2018, tendo a presença de vinte usuários. Temas relacionados à saúde mental, principalmente ansiedade e depressão, são muito prevalentes na população assistida, visto que, em quase todos as consultas de demanda espontânea, existiam pacientes que buscavam atendimento apenas para renovação da prescrição de psicofármacos, sendo resistentes quando se recomendava a cessação dos medicamentos, mesmo quando se esclarecia que os riscos do uso eram maiores que os benefícios. O Brasil apresentou a maior prevalência de transtornos mentais na população adulta, com índices elevados para os transtornos da ansiedade, transtornos do humor e os transtornos relacionados ao uso de substâncias psicoativas ${ }^{20}$.

Observou-se que o grupo referente à temática era o mais relevante na comunidade quanto aos aspectos de adesão e participação. Para falar de saúde mental de maneira eficiente, foi necessário compilar as doenças mais prevalentes como um todo e que geram repercussões mais severas. Foram abordadas a depressão, transtorno de ansiedade generalizada e síndrome do pânico. Frequentemente encontrados na comunidade, os transtornos mentais geram alto custo social e econômico; são universais, pois atingem pessoas de todas as idades, causando incapacitações graves e definitivas que elevam a demanda nos serviços de saúde ${ }^{20}$.

Tendo em vista que essas doenças afetam diretamente a vida de qualquer pessoa que é acometida por elas a tal ponto de gerar uma influência negativa e limitante à realização de suas atividades, todas elas foram esmiuçadas de forma que a população entendesse os aspectos gerais e específicos inerentes a elas, e que tomassem consciência se estavam enfrentando quaisquer dos problemas abordados ou se conheciam alguma pessoa que possivelmente o estivesse, tendo a capacidade de reconhecer, aconselhar e ajudar. Ao falar sobre depressão, foram abordados os aspectos diagnósticos e clínicos, propostas pela quinta edição do Manual de Diagnóstico e Estatístico de Transtornos Mentais (DSM-V), explicitando cada tipo e grau, suas características, como é o comportamento do doente e porque ele é enquadrado em depressivo leve, moderado ou grave, de maneira que foi transmitido para os usuários como de fato a doença se comporta e como ela é manifestada naqueles que são acometidos para que não houvesse dúvidas, e quaisquer que existissem pudessem ser sanadas, gerando neles uma compreensão além de um simples diagnóstico.

A partir disso, foi também exposto o tratamento preconizado para cada tipo de estágio de depressão, tornando ainda mais clara a compreensão acerca dela. Nesse ponto, foram apresentados os fatores essenciais aos cuidados, tanto no âmbito da farmacoterapia quanto da terapia adjuvante, ou seja, aquelas atreladas ao comportamento de maneira mais direta, como o praticar atividades físicas, cuidar da alimentação, participar do grupo de apoio de saúde mental existente na unidade, e esse quesito foi bastante enfatizado devido a sua relação direta com os usuários, e também de realizar terapias cognitivo-comportamentais para maiores chances de se ter um tratamento preciso.

Ao falar sobre ansiedade e síndrome do pânico, sendo a segunda um agravante da primeira situação, da mesma forma foram expostos e abordados os aspectos diagnósticos e clínicos, como essas doenças tem afetado diretamente o dia a dia dos indivíduos nos dias atuais, como identificar se aquilo que o paciente está sentindo de fato configura um quadro patológico ou não, suas comorbidades e porque elas atingem determinada pessoa e como se apresenta. $\mathrm{O}$ tratamento também foi exposto de forma a exaltar a importância de se estar cuidando da mente e que não é o apenas tomar medicamentos que trará uma cura em potencial para o problema, mas sim o prosseguir em cuidar da saúde em âmbito integral, sendo esse o objetivo principal dessa palestra, alertar uma comunidade que apresenta uma considerável parcela da população, cerca de 40 a 50\% deles, fazendo uso de medicamentos controlados e que, nesses casos, não progridem no tratamento por não entender a real importância da doença e que o autocuidado perpassa os comprimidos tomados. Os usuários puderam aprender a ter mais voz ativa diante do seu tratamento e a não serem relapsos quanto a isso por conta da má adesão.

\section{Terceira Atividade}

A terceira palestra, intitulada "O ser mãe, entendendo o pré-natal” foi ministrada no dia 11/04/2018, pela manhã, 
visto que esse era o dia e turno específicos do pré-natal, e contou com a presença de nove gestantes. A unidade assiste um total de doze grávidas. A principal finalidade era tanto incentivar que as usuárias seguissem os planos terapêuticos e intervencionistas propostos pelo pré-natal, sua importância, e porque é necessário realizá-lo e estar sempre em dia com as consultas. O Ministério da Saúde recomenda o número mínimo de seis consultas para uma gestação a termo, com o início do pré-natal no primeiro trimestre e a realização de alguns procedimentos básicos, que incluem exames clínico-obstétricos e laboratoriais, entre outros ${ }^{21}$. De acordo com o Programa de Humanização no Pré-Natal e Nascimento (PHPN), também são preconizadas algumas intervenções qualitativas para melhor adequação ao pré-natal, nas quais orientações sobre amamentação, alimentação suplementar, imunização, entre outras, voltadas às gestantes, são especialmente benéficas ${ }^{21}$.

A palestra foi iniciada comunicando as gestantes o porquê de se realizar o pré-natal, qual a sua finalidade, o que a não realização pode acarretar tanto para a mãe quanto para o bebê em termos de comorbidades e para que ir nas consultas agendadas. O tema foi ministrado em forma de roda de conversa, tornando a atividade mais leve e descontraída, pois sempre que um assunto específico era abordando, antes se questionava a elas a compreensão que tinham a respeito do assunto ou se sabiam algo a respeito, para também entenderem de que forma elas enxergavam a situação e se estavam exercendo seu papel quanto paciente de forma correta. Uma atenção pré-natal de qualidade é capaz de diminuir a morbidade e a mortalidade materno-infantil uma vez que a identificação do risco gestacional pelo profissional permite a orientação e os encaminhamentos adequados em cada momento da gravidez, e sendo assim, fica clara a importância que existe no esclarecimento as mães sobre o realizar o pré-natal, estimulando grupos e gerando nelas a responsabilidade ativa quanto aos seus bebês e sua própria saúde ${ }^{22}$.

Também foi abordado a questão da solicitação dos exames nas consultas, em quais delas é importante pedilos e realiza-los e o que os possíveis resultados indicariam quanto a sua saúde e a do seu bebê, fazendo com que elas ficassem atentas a essas informações e que, quando fossem aos atendimentos daquele momento em diante, se sentissem mais ativas no processo do pré-natal, gerando uma maior interação entre profissional e usuária. Foi percebido que o objetivo principal de alerta e promoção de saúde foi alcançado, tanto pela receptividade das mulheres quanto pela participação e atenção fornecida da parte delas ao que estava sendo discutido. Também foi observado que, quanto mais claras e explícitas as informações eram repassadas, maiores eram as chances tanto de entendimento quanto de futura adesão ao que a unidade propõe no âmbito do pré-natal. Em Camalaú, há uma boa adesão por parte das usuárias quanto ao que se refere a esse assunto e o importante foi sedimentá-lo, estimulá-lo e incentiválo a continuar assim, tendo em vista também o seu aprimoramento.

\section{Quarta Atividade}

Essa atividade foi realizada na manhã do dia 16/04/2018 e intitulada "Conhecendo o funcionamento da USF Camalaú e o que ela tem a oferecer à comunidade". Nessa atividade, foi elucidado aos usuários da USF, presentes na sala de espera, como funciona a unidade e o que ele tinha a oferecer à comunidade, destacando quais os horários de atendimento, os profissionais disponíveis, os grupos de apoio ativos e inativos e a agenda semanal de atendimento de cada profissional vinculado à USF Camalaú. Foram entregues panfletos contendo todas essas informações e explicado cada item presente nesse material.

Desde a implantação do PSF, o Ministério da Saúde propôs uma nova dinâmica para a estruturação dos serviços e ações de saúde, bem como sua relação com a comunidade e entre os diversos níveis de complexidade assistencial $^{5}$. Essa estratégia assume o compromisso de prestar assistência, tanto nas unidades de saúde quanto nos domicílios, identificando os fatores de risco aos quais ela está exposta e neles intervindo de forma apropriada ${ }^{23}$. Essa estratégia ainda propõe-se a humanizar as práticas de saúde, buscando a satisfação do usuário através do estreito relacionamento dos profissionais com a comunidade e estimulando-a ao reconhecimento da saúde como um direito de cidadania e, portanto, expressão de qualidade de vida ${ }^{5}$. Logo, foram expostos aos usuários os direitos e deveres tanto da unidade de saúde e dos profissionais, quanto os da comunidade.

A palestra contou com a presença de vários profissionais da unidade, sendo eles: o médico, a enfermeira, a nutricionista, a fisioterapeuta, a fonoaudióloga, a técnica de enfermagem e de três agentes comunitárias de saúde (ACS). Vale ressaltar que essa palestra foi solicitada pela própria equipe de saúde da USF Camalaú, visto que semanalmente havia discussões na sala de espera devido à falta de informação dos usuários em relação ao horário de funcionamento da unidade e à agenda semanal de cada profissional.

Durante a palestra, em que estavam presentes vinte e cinco usuários, observou-se que muitos deles possuíam dúvidas a respeito dos horários de funcionamento e sobre como era feita a escolha para o atendimento não agendado. Dessa forma, foram explicadas e esclarecidas todas as dúvidas existentes e houve bastante interação do público presente. Os profissionais também aproveitaram a oportunidade para esclarecer suas funções e dar ênfase na importância do autocuidado, evidenciando que a unidade consta de diversos profissionais capacitados para auxiliar nesse processo, sendo necessária apenas a adesão da comunidade. 


\section{Quinta Atividade}

Foi realizada no dia 17/04/2018 (Figura 3), no período da tarde, e intitulada "Principais complicações da Hipertensão Arterial e Diabetes mellitus". Essa palestra teve como objetivo principal esclarecer aos usuários o que pode acontecer com os portadores de hipertensão arterial sistêmica (HAS) e diabetes mellitus (DM) que não realizam um cuidado adequado de suas patologias, sendo evidenciado os grupos de risco para cada complicação. Também foi relatada a importância de se tomar as medicações corretamente, fazer atividades físicas regularmente, ter um acompanhamento regular com os profissionais de saúde da unidade, ter uma dieta equilibrada e individualizada, conforme a patologia presente, dentre outras recomendações.

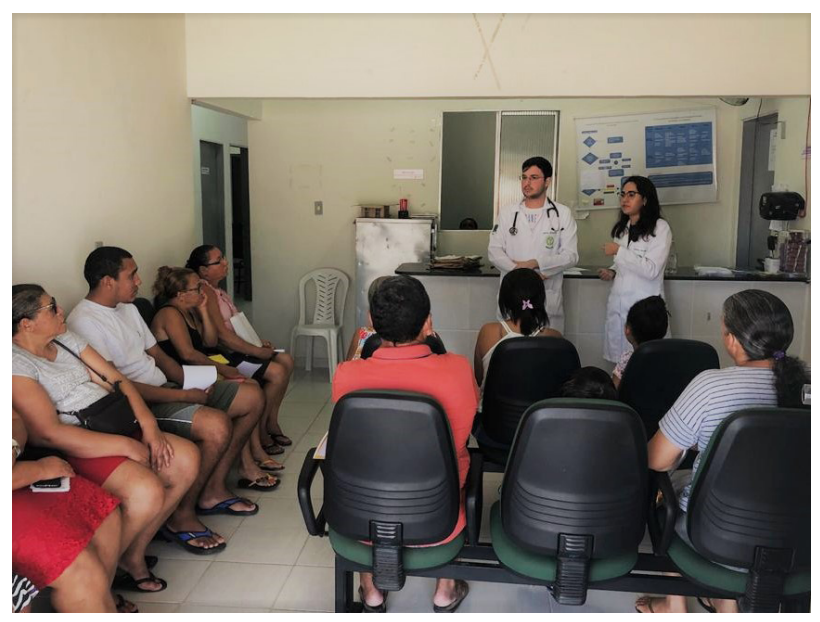

Figura 3. Realização da quinta atividade na USF

Esse tema foi escolhido juntamente com a equipe de saúde da USF Camalaú, visto que essas são as patologias mais frequentes na comunidade e as que possuem menor adesão ao tratamento adequado, ou seja, os usuários da unidade portadores dessas enfermidades não realizam devidamente, e simultaneamente, o tratamento farmacológico e não farmacológico, além de não aderirem ao autocuidado. Vale salientar que essa atividade foi realizada estrategicamente em uma terça-feira, dia dedicado pela unidade para o atendimento de pessoas com DM e HAS, sendo esse o HIPERDIA da USF.

O Programa de Hipertensão Arterial e Diabetes (HIPERDIA) constitui-se em um instrumento de acompanhamento de usuários hipertensos e/ou diabéticos, com as funções de vincular o paciente à Unidade Básica de Saúde (UBS) e à Equipe de Saúde da Família (ESF) de sua referência, realizar uma assistência contínua e de qualidade e fornecer medicamentos de maneira regular. Para tanto, utilizam-se de fichas com vistas a realizar o cadastro e o acompanhamento dos usuários, bem como fazer avaliação de risco entre os pacientes cadastrados ${ }^{24}$.

A HAS e o DM compõem a primeira causa de hospitalizações no sistema público de saúde e são os principais fatores de risco para as doenças cardiovasculares, dos quais cerca de 60 a $80 \%$ dos casos podem ser tratados na rede pública básica ${ }^{25}$. A partir disso, na tentativa de reduzir o número de hospitalizações e de atingir o acompanhamento e o tratamento adequados na atenção básica, diversas estratégias e ações vêm sendo elaboradas e adotadas no Ministério da Saúde ${ }^{25}$. Essas duas doenças apresentam alguns aspectos em comum: etiopatogenia; fatores de risco; tratamento não medicamentoso; caráter crônico; prevenibilidade; assintomaticidade em estágios iniciais; difícil adesão ao tratamento; requisição de acompanhamento por equipe multidisciplinar e fácil diagnóstico ${ }^{26}$.

O diabetes mellitus é a condição crônica que mais cresce, principalmente nos países em desenvolvimento. Destaca-se pela gravidade das suas complicações, além de ser considerado um problema de saúde pública. O DM deve ser investigado em relação às complicações agudas e crônicas e sua relação com o tempo de diagnóstico. As complicações agudas incluem a hipoglicemia, o estado hiperglicêmico hiperosmolar e a cetoacidose diabética. Já as crônicas incluem a retinopatia, nefropatia, cardiopatia isquêmica, neuropatias, doença cerebrovascular e vascular periférica. As degenerativas mais frequentes são o infarto agudo do miocárdio, a arteriopatia periférica, o acidente vascular cerebral e a microangiopatia. As complicações do diabetes aumentam ao longo dos anos, e identificar esta associação pode ser uma estratégia para traçar medidas que minimizem o aparecimento de complicações precocemente $^{27}$.

Já a HAS é apontada como fator de risco para complicações e doenças cardiovasculares na sociedade atual, tais como morte súbita, edema agudo de pulmão, insuficiência renal, infarto agudo do miocárdio (IAM) e acidente vascular encefálico (AVE), explicando $54 \%$ das mortes por acidente vascular encefálico e $47 \%$ daquelas por doença isquêmica do coração. Ademais, a possibilidade de associação da hipertensão arterial sistêmica e do diabetes mellitus é da ordem de $50 \%$, o que, muitas vezes, requer o manejo das duas doenças no mesmo usuário, agravado pelo fato de que sua concomitância potencializa o dano micro e macrovascular decorrente, acarretando alta morbidade cardiocerebrovascular ${ }^{26}$.

Diante do exposto, percebe-se a importância da adesão ao tratamento correto dessas patologias e de um acompanhamento profissional regular, pois a prevenção dessas complicações terá grande impacto na qualidade de vida da população acometida por essas patologias.

Essa palestra contou com a presença de dezenove usuários e foi a que teve maior participação do público, visto que muitos participaram relatando depoimentos de suas próprias vidas e também de pessoas conhecidas que possuíam as complicações das doenças relatadas e que, devido à falta do autocuidado e de um tratamento correto, 
estão atualmente com uma qualidade de vida reduzida e outros, em alguns casos, em estágios terminais. Também foram entregues panfletos contendo o dia e horário do grupo de hipertensos e diabéticos que é disponibilizado pela unidade e foi estimulada a participação regular dos usuários nas atividades desse grupo. Observou-se que os usuários ficaram bem impactados com a palestra e mais motivados para aderirem ao tratamento adequado de suas patologias.

\section{Sexta Atividade}

Essa atividade foi a que finalizou a série de ações planejadas, sendo realizada na tarde do dia 19/04/2018 e intitulada "Autocuidado e saúde". Foi através dela que englobamos todos os temas abordados anteriormente, destacando o que era autocuidado, como ele pode ser feito e sua importância na vida de qualquer pessoa, seja portador de alguma patologia ou não, sendo essa uma importante estratégia de controle e prevenção de doenças e de complicações de patologias já existentes.

Os profissionais da área da saúde vêm se preocupando cada vez mais em relação ao autocuidado de seus pacientes, já que este, muitas vezes, não é incorporado em suas vidas diárias, o que pode resultar em grandes complicações crônicas de suas doenças ${ }^{28}$. A partir disso, percebese a importância do estímulo e incentivo à adesão ao autocuidado, principalmente para os pacientes portadores de doenças crônicas, os quais compõem a maior parte dos usuários da USF Camalaú. Ademais, o autocuidado ainda pode ser uma estratégia importante não apenas na prevenção de complicações de doenças pré-existentes, mas também pode atuar evitando o aparecimento de novas doenças.

Durante a palestra, que contou com a presença de vinte e dois usuários, foram abordadas, de forma didática e objetiva, algumas medidas para a correta execução do autocuidado, foram elas: Ter uma dieta equilibrada e controlar o peso; praticar regularmente exercícios físicos; controlar continuamente a glicemia e pressão arterial; usar corretamente as medicações; ter bons hábitos de higiene pessoal e bucal; cuidar da aparência; ter uma vida social ativa; cessar tabagismo e alcoolismo; investir na espiritualidade; frequentar regularmente um serviço de saúde, como a própria USF, para um acompanhamento especializado e voltado para as reais necessidades; para as mulheres, fazer autoexame das mamas e realizar citológico regularmente, conforme indicação; para os homens acima de 45 anos, realizar anualmente exames preventivos para câncer de próstata; uso de preservativos para evitar doenças sexualmente transmissíveis; ter um bom sono e cuidar da saúde mental. Essas foram as medidas que enfatizadas para se executar eficientemente o autocuidado.

Também foi reforçada a existência de grupos de apoio na USF que poderão auxiliar nesse autocuidado, como o grupo de saúde mental, de exercícios físicos e o de hipertensão arterial e diabetes. A palestra contou com a presença do médico da unidade, tendo sido sua participação fundamental para estimular os usuários a terem hábitos saudáveis e utilizarem o que a unidade tem a oferecer de forma gratuita e com o acompanhamento por profissionais qualificados.

\section{CONCLUSÃO}

Pôde-se observar que houve melhora na adesão ao autocuidado nos usuários da USF Camalaú, que foram questionados após a execução das ações, e que essas atividades foram imprescindíveis para isso. Esse estudo proporcionou a criação de vínculo com a comunidade e isso facilitou a interação com os pacientes, promovendo um melhor planejamento da terapêutica. Durante os atendimentos efetuados após a realização das atividades, as condutas tinham um nível de aceitabilidade bem maior quando comparadas às consultas em que não era realizada uma ação anteriormente. Essa intervenção, a longo prazo, poderá contribuir para uma melhora significativa na terapêutica dos usuários, no controle de suas patologias, na prevenção de doenças e complicações e, principalmente, favorecer uma melhor qualidade de vida para os usuários.

A realização desse estudo evidenciou, no âmbito profissional, a importância da educação em saúde e como atividades como essas devem ser realizadas pelos profissionais para que a comunidade tenha uma melhor aceitação do manejo terapêutico escolhido e isso contribuirá para uma atuação profissional diferenciada, que visa difundir o conhecimento e não restringi-lo. Também mostrou que a interação da equipe de saúde da família é essencial para que a USF possa assegurar integralmente a promoção à saúde e evidenciou a importância das reuniões de equipe neste processo.

Ademais, proporcionou a criação de vínculo com a comunidade, o que facilitou a interação com os pacientes e promoveu certa segurança, por parte dos usuários, durante o planejamento da terapêutica. Vale ressaltar que as informações repassadas durante as atividades devem ser reforçadas em cada atendimento realizado pelos profissionais e integrantes da equipe de saúde da unidade, para que uma maior parcela da população esteja ciente dos benefícios gerados pelo autocuidado e sua importância na melhora da qualidade de vida.

Agradecimentos: Agradecemos a Deus, em primeiro lugar, que é digno de toda honra. Também agradecemos a toda equipe e usuários da USF Camalaú, em especial a Dr. Diógenes e Sâmia, além da FCM-PB que nos ofereceu o campo de prática para elaboração do artigo.

Participação no artigo: Sousa GF e Oliveira KDP - contribuíram na concepção e realização dos estudos, na coleta, compilação e interpretação dos dados e na escrita do manuscrito. Queiroz SMD - orientou, ajudou na interpretação dos resultados, fez revisão e correção do manuscrito. 


\section{REFERÊNCIAS}

1. Silva IJ, Oliveira MFV, Silva SED, Polaro SHI, Radünz V, Santos EKA, Santana ME. Cuidado, autocuidado e cuidado de si: uma compreensão paradigmática para o cuidado de enfermagem. Rev Esc Enferm USP. 2009;43(3):697-703. doi: http://dx.doi.org/10.1590/S0080-62342009000300028.

2. Chubaci RYS, Fraga IM. As motivações para o autocuidado dos docentes de uma universidade pública: um enfoque da fenomenologia social. Rev Kairós. 2013;16(2):167-90. Disponível em: https://revistas.pucsp.br/kairos/article/ view/17638/13137.

3. Brasil. Ministério da Saúde. Secretaria de Políticas de Saúde. Programa Saúde da Família. Brasília (DF); 1997. Disponível em: http://bvsms.saude.gov.br/bvs/publicacoes/cd09_16.pdf.

4. Brasil. Ministério da Saúde. Manual para organização da atenção básica. Brasília (DF); 1999. Disponível em: http:// bvsms.saude.gov.br/bvs/publicacoes/organizacao_atencao.pdf.

5. Sousa MF. A enfermagem reconstruindo sua prática: mais que uma conquista no PSF. Rev Bras Enferm. 2000;53(n. esp.):25-30. doi: http://dx.doi.org/10.1590/S003471672000000700004 .

6. Freire P. Pedagogia da autonomia. São Paulo: Paz e Terra; 1997.

7. Freire P. Educação como prática da liberdade. São Paulo: Paz e Terra; 2002.

8. Freire P. Pedagogia da esperança: um reencontro com a pedagogia do oprimido. Rio de Janeiro: Paz e Terra; 2003.

9. Sampaio J, Santos GC, Agostini M, Salvador AS. Limites e potencialidades das rodas de conversa no cuidado em saúde: uma experiência com jovens no sertão pernambucano. Interface (Botucatu). 2014;18(Suppl 2):1299-11. doi: http:// dx.doi.org/10.1590/1807-57622013.0264.

10. Moura AF, Lima MG. A reinvenção da roda: roda de conversa: um instrumento metodológico possível. Rev Temas Educ. 2014;23(1):98-106. Disponível em: http://www.periodicos. ufpb.br/index.php/rteo/article/view/18338/11399.

11. Brasil. Ministério da Saúde. Secretaria de Atenção à Saúde. Departamento de Atenção Básica. Estratégias para o cuidado da pessoa com doença crônica. Cadernos de Atenção Básica, n. 35. Brasília (DF); 2014. Disponível em: http://bvsms.saude. gov.br/bvs/publicacoes/estrategias cuidado pessoa doenca cronica_cab35.pdf.

12. Donabedian A. The definition of quality and approaches to its assessment. Ann Arbor (MI): Health Administration Press; 1980.

13. Leite SN, Vieira M, Veber AP. Estudos de utilização de medicamentos: uma síntese de artigos publicados no Brasil e América Latina. Cienc Saúde Coletiva. 2008;13(Suppl):793-802. doi: http://dx.doi.org/10.1590/ S1413-81232008000700029.

14. Pereira MR, Coutinho MSSA, Freitas PF, D'Orsi E, Bernardi A, Hass R. Prevalência, conhecimento, tratamento e controle de hipertensão arterial sistêmica na população adulta urbana de Tubarão, Santa Catarina, Brasil, em 2003. Cad Saúde Pública. 2007;23(10):2363-74. doi: http://dx.doi.org/10.1590/ S0102-311X2007001000011.

15. Portela MC, Lima SML, Martins M, Travassos C. Ciência da melhoria do cuidado de saúde: bases conceituais e teóricas para a sua aplicação na melhoria do cuidado de saúde. Cad Saúde Pública. 2016;32(Suppl 2):e00105815. doi: http:// dx.doi.org/10.1590/0102-311X00105815.
16. Alvarez SQ, Gomes GC, Oliveira AMN, Xavier DM. Grupo de apoio/ suporte como estratégia de cuidado: importância para familiares de usuários de drogas. Rev Gaúcha Enferm. 2012;33(2):102-8. doi: http://dx.doi.org/10.1590/S198314472012000200015.

17. Beraldo AA, Andrade RLP, Orfão NH, Silva-Sobrinho RA, Pinto ESG, Wysocki AD, Brunello MEF, Monroe AA, Scatena LM, Villa TCS. Adesão ao tratamento da tuberculose na Atenção Básica: percepção de doentes e profissionais em município de grande porte. Esc Anna Nery. 2017;21(4):e20170075. doi: http://dx.doi.org/10.1590/21779465-EAN-2017-0075.

18. Nogueira AF, Facchinetti V, Souza MVN, Vasconcelos TRA. Tuberculose: uma abordagem geral dos principais aspectos. Rev Bras Farm. 2012;93(1):3-9. Disponível em: http://www. rbfarma.org.br/files/rbf-2012-93-1-1.pdf.

19. Melo RHV, Felipe MCP, Cunha ATR, Vilar RLA, Pereira EJS, Carneiro NEA, Freitas NGHB, Júnior JD. Roda de conversa: uma articulação solidária entre ensino, serviço e comunidade. Rev Bras Educ Med. 2016;40(2):301-9. doi: http://dx.doi. org/10.1590/1981-52712015v40n2e01692014.

20. Santos EG, Siqueira MM. Prevalência dos transtornos mentais na população adulta brasileira: uma revisão sistemática de 1997 a 2009. J Bras Psiquiatr. 2010;59(3):238-46. doi: http:// dx.doi.org/10.1590/S0047-20852010000300011.

21. Nunes JT, Gomes KRO, Rodrigues MTP, Mascarenhas MDM. Qualidade da assistência pré-natal no Brasil: revisão de artigos publicados de 2005 a 2015. Cad Saúde Coletiva. 2016;24(2):252-61. doi: http://dx.doi.org/10.1590/1414462X201600020171.

22. Tomasi E, Fernandes PAA, Fischer T, Siqueira FCV, Silveira DS, Thumé E, Duro SMS, Saes MO, Nunes BP, Fassa AG, Facchini LA. Qualidade da atenção pré-natal na rede básica de saúde do Brasil: indicadores e desigualdades sociais. Cad Saúde Pública. 2017;33(3):e00195815. doi: http://dx.doi. org/10.1590/0102-311x00195815.

23. Silva CC, Silva ATMC, Losing A. A integração e articulação entre as ações de saúde e de educação no Programa de Saúde da Família-PSF. Rev Eletr Enferm. 2006;8(1):70-4. Disponível em: https://revistas.ufg.br/fen/article/view/941/1147.

24. Brasil. Ministério da Saúde. Secretaria de Atenção à Saúde. Departamento de Atenção Básica. Plano de Reorganização da Atenção à Hipertensão Arterial e ao Diabetes Mellitus no Brasil. Brasília (DF); 2001. Disponível em: http://bvsms. saude.gov.br/bvs/publicacoes/miolo2002.pdf.

25. Silva JVM, Mantovani MF, Kalinke LP, Ulbrich EM. Avaliação do Programa de Hipertensão Arterial e Diabetes Mellitus na visão dos usuários. Rev Bras Enferm. 2015;68(4):626-32. doi: http://dx.doi.org/10.1590/0034-7167.2015680408i.

26. Santos JC, Moreira TMM. Fatores de risco e complicações em hipertensos/diabéticos de uma regional sanitária do nordeste brasileiro. Rev Esc Enferm USP. 2012;46(5):1125-32. doi: http://dx.doi.org/10.1590/S0080-62342012000500013.

27. Cortez DN, Reis IA, Souza DA, Macedo MM, Torres HC. Complicações e o tempo de diagnóstico do diabetes mellitus na atenção primária. Acta Paul Enferm. 2015; 28(3):250-5. doi: http://dx.doi.org/10.1590/1982-0194201500042.

28. Orozco LB, Alves SHS. Diferenças do autocuidado entre pacientes com diabetes mellitus tipo 1 e 2 . Psicol Saúde Doenças. 2017;18(1):234-47. doi: http://dx.doi. org/10.15309/17psd180119.

Recebido: 10.11 .18

Aceito: 19.02.19 